\title{
Incidence of anemia in patients diagnosed with solid tumors receiving chemotherapy, 20I0-20I3
}

This article was published in the following Dove Press journal:

Clinical Epidemiology

18 April 2016

Number of times this article has been viewed

\section{Hairong $X u^{\prime}$ \\ Lanfang $\mathrm{Xu}^{2}$ \\ John H Page' \\ Kim Cannavale ${ }^{2}$ \\ Olivia Sattayapiwat ${ }^{2}$ \\ Roberto Rodriguez ${ }^{3}$ \\ Chun $\mathrm{Chao}^{2}$}

'Center for Observational Research, Amgen Inc., Thousand Oaks, CA, USA; ${ }^{2}$ Department of Research and Evaluation, Kaiser Permanente Southern California, Pasadena, CA, USA; ${ }^{3}$ Department of Hematology Oncology, Los Angeles Medical Center, Kaiser Permanente Southern California, Psadena, CA, USA
Correspondence: Hairong Xu Center for Observational Research, Amgen Inc., One Amgen Center Drive, Mailstop 24-2-A, Thousand Oaks, CA 91320 , USA

Tel +I 805447 I523

Email hairongx@amgen.com
Purpose: The purpose of this study was to evaluate and characterize the risk of anemia during the course of chemotherapy among patients with five common types of solid tumors.

Patients and methods: Patients diagnosed with incident cancers of breast, lung, colon/ rectum, stomach, and ovary who received chemotherapy were identified from Kaiser Permanente Southern California Health Plan (2010-2012). All clinical data were collected from the health plan's electronic medical records. Incidence proportions of patients developing anemia and $95 \%$ confidence intervals were calculated overall and by anemia severity and type, as well as by stage at cancer diagnosis, and by chemotherapy regimen and cycle.

Results: A total of 4,426 patients who received chemotherapy were included. Across cancers, $3,962(89.5 \%)$ patients developed anemia during the course of chemotherapy (normocytic $85 \%$, macrocytic $10 \%$, microcytic 5\%; normochromic $47 \%$, hyperchromic $44 \%$, hypochromic $9 \%$ ). The anemia grades were distributed as follows: $58 \%$ were grade $1,34 \%$ grade $2,8 \%$ grade 3 , and $<1 \%$ grade 4 . The incidence of grade $2+$ anemia ranged from $26.3 \%$ in colorectal cancer patients to $59.2 \%$ in ovarian cancer patients. Incidence of grade $2+$ anemia increased from $29 \%$ in stage I to $49 \%$ in stage IV. Incidence of grade $2+$ anemia varied from $18.2 \%$ in breast cancer patients treated with cyclophosphamide + docetaxel regimen to $59.7 \%$ in patients with ovarian cancer receiving carboplatin + paclitaxel regimen.

Conclusion: The incidence of moderate-to-severe anemia (hemoglobin $<10 \mathrm{~g} / \mathrm{dL}$ ) remained considerably high in patients with solid tumors receiving chemotherapy. The risk of anemia was greater in patients with distant metastasis.

Keywords: solid tumors, anemia, chemotherapy, incidence

\section{Introduction}

Myelosuppressive chemotherapy is a primary treatment modality for treating cancer, since in some settings this strategy decreases the risk of tumor reoccurrence and mortality. Cytotoxic chemotherapy agents can induce anemia through directly impairing hematopoiesis, including synthesis of red blood cell (RBC) precursors in the bone marrow; decreasing renal production of erythropoietin; or a combination of both. ${ }^{1,2}$

The risk and severity of anemia during chemotherapy vary significantly depending on the type, schedule, and intensity of treatments, and whether the patient has received previous myelosuppressive chemotherapy or radiation therapy, as well as the patient's underlying medical conditions. ${ }^{3}$ Previous studies reported that the incidence of anemia may range from $20 \%$ to $60 \%$ at the time of cancer diagnosis and reach as high as 60-90\% during cancer treatments. ${ }^{4-7}$ Groopman and Itri ${ }^{5}$ conducted a comprehensive review of published clinical trials of the most common single agents and combination 
chemotherapy regimens. Risk reported in clinical trials, however, may not reflect real-world clinical practice. Due to many of the enrollment exclusionary criteria applied in randomized clinical trials, patients in real-world clinical practice may have, on average, higher anemia risks due to a higher prevalence of comorbidities, older age, and poorer performance status. In addition, the anemia risk profile may have changed given the changes in current oncology practice.

Anemia can cause fatigue, drowsiness, depression, dyspnea, tachycardia, and dizziness, which may result in delay to subsequent cycles of chemotherapy or have a negative impact on patients' quality of life. ${ }^{8-10}$ Before the 1990 s, RBC transfusions were the primary treatment for severe or lifethreatening anemia. Since then, erythropoiesis-stimulating agents (ESAs) have become available for correcting mildto-moderate anemia and preventing severe anemia. However, beginning in 2003, some studies have suggested that ESA use was associated with decreased patient survival and/or tumor progression or recurrence in patients with breast, lymphoid, cervical, head and neck, and non-small-cell lung cancers. ${ }^{1-14}$ These data led to a series of prescribing information and policy changes. ${ }^{15}$ Following these changes, there is evidence of decreased use of ESAs, coupled with a lowering of the average hemoglobin $(\mathrm{Hb})$ concentration among cancer patients receiving myelosuppressive chemotherapy treatment. ${ }^{16}$ While ESAs are not indicated as a substitute for packed $\mathrm{RBC}$ transfusions in patients who require immediate correction of anemia, the potential shift in the perceived $\mathrm{Hb}$ trigger levels for anemia treatment has likely resulted in an overall increase in the risk and severity of anemia.

Given that little data exist on the risk of anemia among patients receiving chemotherapy in current oncology practice, we conducted this retrospective cohort study to comprehensively evaluate and characterize the risk of chemotherapyinduced anemia (CIA) during the course of chemotherapy among patients with five common types of solid tumors diagnosed in 2010-2012 at Kaiser Permanente Southern California (KPSC).

\section{Patients and methods}

\section{Study setting and data sources}

KPSC is an integrated managed care organization that provides comprehensive health services for 3.5 million racially/ethnically and socioeconomically diverse enrollees who are broadly representative of residents in southern California. ${ }^{17}$ KPSC maintains a number of electronic clinical records for virtually all aspects of care delivered, including diagnoses in the form of International Statistical Classification of Disease (ICD-9) codes, medical procedures, pharmacy dispensing, laboratory test results, and disease registries, all linkable through a unique member identifier. Patients diagnosed with incident breast, lung, gastric, ovarian, or colorectal cancer were identified using KPSC's Surveillance, Epidemiology, and End Results-affiliated cancer registry. KPSC's cancer registry routinely collects information on age at diagnosis, sex, race/ethnicity, histology, location, stage, and initial cancer treatment. The quality of the cancer registry data is assured by the Surveillance, Epidemiology, and End Results standard. Ethical approval from KPSC review board was obtained for this study.

\section{Study population}

The study cohort comprised patients who met the following inclusion criteria: 1) diagnosed with incident breast, lung, gastric, ovarian, or colorectal cancer at age 18 or older at KPSC between March 2010 and December 2012; 2) initiated chemotherapy at KPSC before June 30, 2013; 3) had at least one laboratory measurement of $\mathrm{Hb}$ during chemotherapy; and 4) had at least 12 months of KPSC membership prior to cancer diagnosis. We further excluded patients who had at least one of the following criteria: 1) had unknown cancer stage or for whom chemotherapy agents could not be identified; 2) had a diagnosis of myelodysplastic syndromes or inherited anemia; 3) had an $\mathrm{Hb}$ concentration $<10 \mathrm{~g} / \mathrm{dL}$ within 3 months prior to chemotherapy initiation; 4) had a transfusion within 2 weeks prior to chemotherapy initiation; 5) received bone marrow or stem cell transplantation within 12 months prior to chemotherapy initiation or during the chemotherapy course; or 6) received radiation therapy within 4 months prior to chemotherapy initiation.

\section{Chemotherapy regimens and cycles}

For each patient, the first observed course of chemotherapy was characterized, as was each cycle within that course; only the first course of chemotherapy was considered in analyses. The index date was defined as the date of chemotherapy initiation during the study period. Patients were followed from the index date to 60 days after their last dose of first-course chemotherapy, death, or end of KPSC membership, whichever came first. Chemotherapy regimens and cycles were characterized from the electronic oncology pharmacy dispensing databases at KPSC. The oncology pharmacy dispensing databases contain oncology treatment information for all KPSC cancer patients, including information on chemotherapy, ancillary therapy, and biologics. Data entered by KPSC pharmacists and nurses encompass generic and brand names of the chemotherapy regimens, dosages, dates of administration, and start/end dates of chemotherapy cycles. 


\section{Anemia measurement}

Following National Cancer Institute's Common Terminology Criteria for Adverse Events, anemia was classified as grade 1: $10 \mathrm{~g} / \mathrm{dL}$ to lower limit of normal $(14 \mathrm{~g} / \mathrm{dL}$ for men and $12 \mathrm{~g} / \mathrm{dL}$ for women); grade $2: 8.0-9.9 \mathrm{~g} / \mathrm{dL}$; grade $3: 6.5-7.9 \mathrm{~g} / \mathrm{dL}$; and grade $4:<6.5 \mathrm{~g} / \mathrm{dL} .{ }^{5}$ Most severe anemia grade was used to estimate the incidence proportion of anemia by grade. We also determined the type of anemia using mean corpuscular volume (MCV) and mean corpuscular hemoglobin (MCH) as microcytic anemia (MCV $<80 \mathrm{fL}$ ), normocytic anemia $(80 \leq \mathrm{MCV} \leq 100 \mathrm{fL})$, macrocytic anemia (MCV >100 fL), hypochromic anemia $(\mathrm{MCH}<27 \mathrm{pg} /$ cell), normochromic anemia $(27 \leq \mathrm{MCH} \leq 31 \mathrm{pg} / \mathrm{cell})$, and hyperchromic anemia $(\mathrm{MCH}>31 \mathrm{pg} / \mathrm{cell})^{2}$

\section{Covariates}

Patient characteristics that were examined in the study included age at cancer diagnosis, sex, race/ethnicity, cancer stage at diagnosis, length of KPSC membership, and presence of selected comorbid conditions. Chronic comorbidities were assessed from the beginning of the 12-month pretreatment period to the first day of the chemotherapy course.

\section{Statistical analysis}

Characteristics of patients and their treatment were described, and categorical variables were reported as percentages; for continuous variables, means, standard deviations, medians, and selected quartiles were reported. The incidence proportions of anemia and corresponding $95 \%$ confidence intervals were estimated overall and by severity and type, as well as by cancer stage at diagnosis, and by regimen and cycle. We also estimated the incidence proportions of anemia by age, sex, and ethnicity; and the results were similar across subgroups (data not shown). In addition, we estimated the regimen-specific incidence proportions of anemia for the common regimens ( $\mathrm{n}>100$ patients). To minimize the selection bias and have a more representative longitudinal profile of anemia risk over chemotherapy cycles, we estimated the cycle-specific incidence proportion up to the number of cycles with at least $50 \%$ of patients remaining in the sample (less than $50 \%$ of loss to follow-up). All analyses were conducted using SAS statistical software version 9.2 (Statistical Analysis System Inc., Cary, NC, USA).

\section{Results}

The study sample included 4,426 patients who met the specified inclusion criteria (Figure 1). Their baseline characteristics are summarized in Table 1. Of the five cancers of interest,

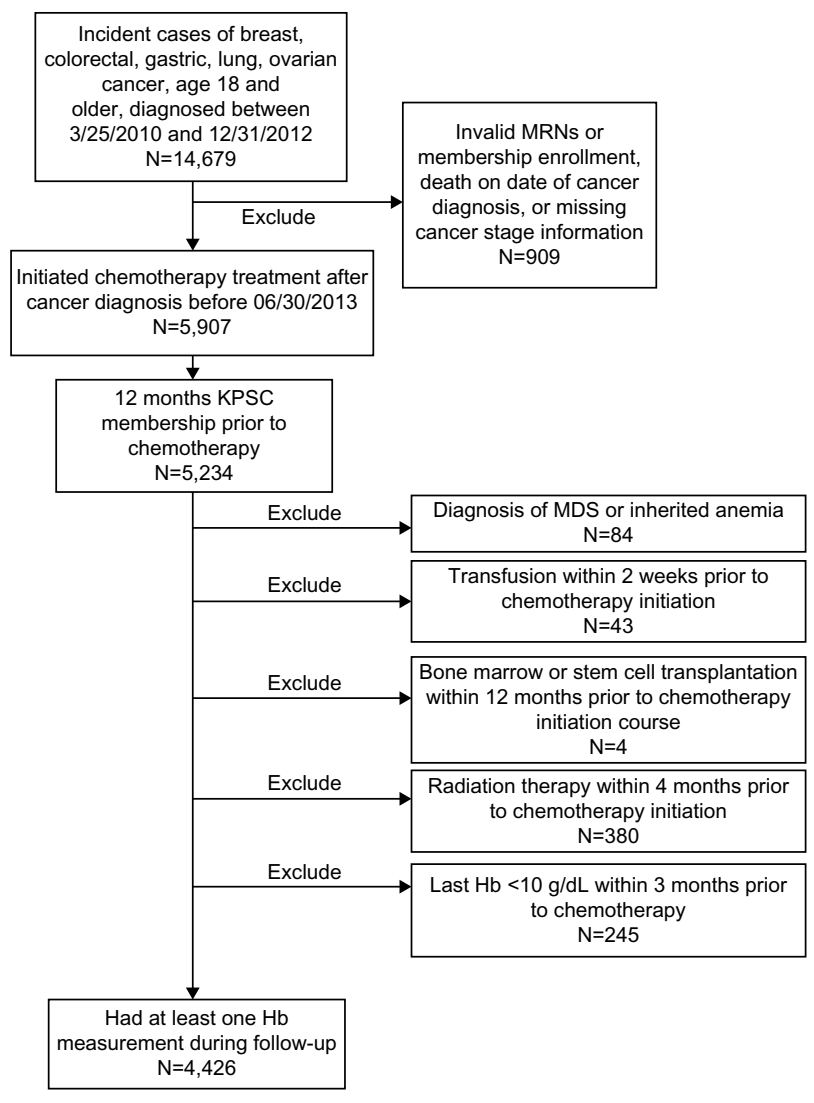

Figure I Study Population-inclusion/exclusion flow diagram. Abbreviations: KPSC, Kaiser Permanente Southern California; MRN, member registration number; MDS, myelodysplastic syndrome; $\mathrm{Hb}$, hemoglobin.

breast cancer was the most frequent diagnosis $(n=2,348$, $53.1 \%)$, followed by lung cancer $(\mathrm{n}=888,20.1 \%)$, colorectal cancer $(n=678,15.3 \%)$, ovarian cancer $(n=319,7.2 \%)$, and gastric cancer $(\mathrm{n}=193,4.4 \%)$. Overall, mean age (standard deviation) was 59.3 (11.9) years. There were numerically more females than males. The majority of patients were diagnosed with stage III or IV disease, except those with breast cancer, in whom stage I to II cancers predominated. The mean (standard deviation) $\mathrm{Hb}$ before chemotherapy was 12.9 (1.3) $\mathrm{g} / \mathrm{dL}$ across cancer types. The most common comorbid conditions before starting chemotherapy were hypertension (42\%), diabetes (18\%), and chronic obstructive pulmonary disease/emphysema (14\%).

Across cancers, 3,962 (89.5\%) patients developed any grade anemia during the course of chemotherapy, ranging from $86.3 \%$ in patients diagnosed with breast cancer to $98.4 \%$ in ovarian cancer patients. Of these 3,962 anemic patients, $58 \%, 34 \%, 8 \%$, and $<1 \%$ had grades $1,2,3$, and 4 anemia as the most severe grade during the study period, respectively; $85 \%, 10 \%$, and $5 \%$ had normocytic, macrocytic, and microcytic anemia, respectively; and $47 \%, 44 \%$, and $9 \%$ of patients had normochromic, hyperchromic, and 
Table I Characteristics of the study population

\begin{tabular}{|c|c|c|c|c|c|c|}
\hline & $\begin{array}{l}\text { Total } \\
(\mathrm{N}=\mathbf{4 , 4 2 6 )}\end{array}$ & $\begin{array}{l}\text { Breast } \\
(\mathrm{N}=2,348)\end{array}$ & $\begin{array}{l}\text { Colorectal } \\
(\mathrm{N}=678)\end{array}$ & $\begin{array}{l}\text { Gastric } \\
(N=193)\end{array}$ & $\begin{array}{l}\text { Lung } \\
(\mathbf{N}=\mathbf{8 8 8})\end{array}$ & $\begin{array}{l}\text { Ovary } \\
(N=319)\end{array}$ \\
\hline $\begin{array}{l}\text { Age at cancer diagnosis (years), } \\
\text { mean (SD) }\end{array}$ & $59.3(11.9)$ & $55.9(\mathrm{II} .2)$ & $59.9(11.7)$ & $61.4(12.1)$ & $66.8(9.8)$ & $61.1(12.5)$ \\
\hline Male, n (\%) & $978(22.1)$ & $19(0.8)$ & $349(5 \mid .5)$ & $132(68.4)$ & $478(53.8)$ & $0(0)$ \\
\hline BMI $\left(\mathrm{kg} / \mathrm{m}^{2}\right)$, mean $(\mathrm{SD})$ & $28.1(6.3)$ & $29.3(6.7)$ & $27.7(5.7)$ & $26.1(5.1)$ & $26.2(5.2)$ & $26.4(6.4)$ \\
\hline \multicolumn{7}{|l|}{ Race/ethnicity, n (\%) } \\
\hline White & $2,352(53.1)$ & $\mathrm{I}, 160(49.4)$ & $360(53.1)$ & $84(43.5)$ & $57 \mid(64.3)$ & $177(55.5)$ \\
\hline Black & $552(12.5)$ & $316(13.5)$ & $90(13.3)$ & $15(7.8)$ & $106(11.9)$ & $25(7.8)$ \\
\hline Hispanic & $937(21.2)$ & $536(22.8)$ & $153(22.6)$ & $74(38.3)$ & $106(11.9)$ & $68(21.3)$ \\
\hline Asian/Pacific Islander & $565(12.8)$ & $328(15.0)$ & $72(10.6)$ & $20(10.4)$ & $99(11.2)$ & $46(14.4)$ \\
\hline Other/unknown & $20(0.5)$ & $8(0.3)$ & $3(0.4)$ & $0(0)$ & $6(0.7)$ & $3(0.9)$ \\
\hline \multicolumn{7}{|l|}{ Cancer stage at diagnosis, $\mathrm{n}(\%)$} \\
\hline 1 & $882(19.9)$ & $732(31.2)$ & $25(3.7)$ & $17(8.8)$ & $40(4.5)$ & $68(21.3)$ \\
\hline II & $\mathrm{I}, 384(3 \mathrm{I} .3)$ & $\mathrm{I}, \mathrm{II} \mathrm{I}(47.3)$ & $109(16.1)$ & $27(14.00)$ & $82(9.2)$ & $55(17.2)$ \\
\hline III & $\mathrm{I}, 199(27.1)$ & $415(17.7)$ & $381(56.2)$ & $64(33.2)$ & $247(27.8)$ & $92(28.8)$ \\
\hline IV & $961(21.7)$ & $90(3.8)$ & $163(24.0)$ & $85(44.0)$ & $519(58.5)$ & $104(32.6)$ \\
\hline \multicolumn{7}{|c|}{ Length of KPSC membership prior to chemotherapy (year) } \\
\hline Mean (SD) & I5.3 (I2.0) & | $4.6(\mid 1.7)$ & $14.8(\mid 1.7)$ & $16.0(11.8)$ & I7.I (I2.7) & $16.2(12.6)$ \\
\hline Median (QI, Q3) & $12.3(5.2,22.6)$ & $11.5(4.9,21.8)$ & $12.0(4.9,22.3)$ & $13.7(6.3,23.6)$ & $14.4(6.5,25.2)$ & $13.7(5.5,23.2)$ \\
\hline \multicolumn{7}{|l|}{ Number of chemotherapy cycles } \\
\hline Mean (SD) & $7.0(5.0)$ & $8.0(5.4)$ & $8.4(5.2)$ & $4.1(2.4)$ & $4.2(3.0)$ & $6.0(2.5)$ \\
\hline Median (QI, Q3) & $6.0(4.0,8.0)$ & $6.0(4.0,8.0)$ & $8.0(5.0,12.0)$ & $4.0(2.0,6.0)$ & $4.0(2.0,6.0)$ & $6.0(6.0,6.0)$ \\
\hline \multicolumn{7}{|l|}{ Baseline $\mathrm{Hb}(\mathrm{g} / \mathrm{dL})$} \\
\hline Mean (SD) & $12.9(1.3)$ & I3.I (I.I) & $12.4(1.3)$ & $12.5(1.6)$ & $13.0(\mid .5)$ & $12.0(I . I)$ \\
\hline Median (QI, Q3) & $12.9(12.0,13.7)$ & $13.2(12.4,13.8)$ & I2.4 (I I.4, I3.3) & I2.4 (I I.4, I3.5) & $\mid 3.0(|| .9, \mid 4.0)$ & $12.0(\mid 1.2,12.8)$ \\
\hline \multicolumn{7}{|c|}{ History of other conditions/events prior to chemotherapy course, n (\%) } \\
\hline Surgery & $3,360(75.9)$ & $2,062(87.8)$ & $577(85.1)$ & $90(46.6)$ & $367(4 \mid .3)$ & $264(82.8)$ \\
\hline Congestive heart failure & $83(1.9)$ & $25(1.1)$ & $15(2.2)$ & $2(1.0)$ & $36(4.1)$ & $5(1.6)$ \\
\hline COPD/emphysema & $628(14.2)$ & $212(9.0)$ & $53(7.8)$ & $8(4.2)$ & $329(37.1)$ & $26(8.2)$ \\
\hline Diabetes & $795(18.0)$ & $359(15.3)$ & I5I (22.3) & $43(22.3)$ & $192(21.6)$ & $50(15.7)$ \\
\hline Diabetes with end-organ damage & $435(9.8)$ & $176(7.5)$ & $85(12.5)$ & $31(16.1)$ & $120(13.5)$ & $23(7.2)$ \\
\hline Liver disease & $66(1.5)$ & $23(1.0)$ & $14(2.1)$ & $7(3.6)$ & $14(1.6)$ & $8(2.5)$ \\
\hline Renal disease & $352(8.0)$ & $120(5.1)$ & $72(10.6)$ & $20(10.4)$ & $120(13.5)$ & $20(6.3)$ \\
\hline Rheumatoid disease & $65(1.5)$ & $33(1.4)$ & $5(0.7)$ & I (0.5) & $16(1.8)$ & $10(3.1)$ \\
\hline Thyroid disorder & $491(11.1)$ & $275(11.7)$ & $52(7.7)$ & $20(10.4)$ & $93(10.5)$ & $51(16.0)$ \\
\hline Hypertension & $\mathrm{I}, 872(42.3)$ & $852(36.3)$ & $290(42.8)$ & $86(44.6)$ & $501(56.4)$ & $143(44.8)$ \\
\hline Myocardial infarction & $104(2.4)$ & $17(0.7)$ & $20(3.0)$ & $4(2.1)$ & $59(6.6)$ & $4(1.3)$ \\
\hline Osteoarthritis & $386(8.7)$ & $193(8.2)$ & $55(8.1)$ & $15(7.8)$ & $88(9.9)$ & $35(11.0)$ \\
\hline Peripheral vascular disease & $128(2.9)$ & $17(0.7)$ & $2 I(3 . I)$ & $3(1.6)$ & $80(9.0)$ & $7(2.2)$ \\
\hline Thrombosis & $44(1.0)$ & $7(0.3)$ & $15(2.2)$ & $4(2.1)$ & $12(1.4)$ & $6(1.9)$ \\
\hline Peptic ulcer disease & $35(0.8)$ & $5(0.2)$ & $2(0.3)$ & I5 (7.8) & $12(1.4)$ & $\mathrm{I}(0.3)$ \\
\hline HIV infection & $6(0.1)$ & $\mathrm{I}(0.0)$ & $3(0.4)$ & I $(0.5)$ & $\mathrm{I}(0.1)$ & $0(0.0)$ \\
\hline
\end{tabular}

Abbreviations: KPSC, Kaiser Permanente Southern California; BMI, body mass index; COPD, chronic obstructive pulmonary disease; Hb, hemoglobin; HIV, human immunodeficiency virus; SD, standard deviation.

hypochromic anemia, respectively. The incidence proportion of grade $2+$ anemia $(\mathrm{Hb}<10 \mathrm{~g} / \mathrm{dL})$ was highest in patients with ovarian cancer (59.2\%) and lowest in patients with colorectal cancer $(26.3 \%)$. The risk of severe anemia (grade $3+, \mathrm{Hb}<8 \mathrm{~g} / \mathrm{dL}$ ) was highest in patients with ovarian cancer $(16.9 \%)$ and lowest in patients with colorectal cancer (4\%) (Table 2).

Table 3 lists the distribution of chemotherapy regimens by tumor type. For breast cancer patients, the most common regimens were docetaxel + cyclophosphamide (TC; 36\%), doxorubicin + cyclophosphamide followed by paclitaxel/docetaxel ( $\mathrm{AC} \rightarrow \mathrm{T} ; 28 \%$ ), and docetaxel + carboplatin + trastuzumab (TCH: 17\%). The most common regimens among patients with lung cancer were carboplatin + paclitaxel (34.1\%), carboplatin + etoposide $(23.5 \%)$, and pemetrexed + cisplatin/carboplatin (18.2\%).

The incidence of any grade anemia varied significantly for selected chemotherapy regimens, ranging from $77 \%$ to $96 \%$ (Table 4 ). The incidence of grade $2+$ anemia varied from $18.2 \%$ in breast cancer patients treated with TC regimen to $59.7 \%$ in patients with ovarian cancer receiving carboplatin + paclitaxel (Carbo-Tax; National Cancer 
Table 2 Proportion of patients developing CIA by CIA severity and type

\begin{tabular}{|c|c|c|c|c|c|c|}
\hline & $\begin{array}{l}\text { Total } \\
(\mathrm{N}=\mathbf{4 , 4 2 6 )}\end{array}$ & $\begin{array}{l}\text { Breast } \\
(\mathrm{N}=2,348)\end{array}$ & $\begin{array}{l}\text { Colorectal } \\
(\mathrm{N}=678)\end{array}$ & $\begin{array}{l}\text { Gastric } \\
(\mathrm{N}=193)\end{array}$ & $\begin{array}{l}\text { Lung } \\
(\mathrm{N}=\mathbf{8 8 8})\end{array}$ & $\begin{array}{l}\text { Ovary } \\
(\mathrm{N}=319)\end{array}$ \\
\hline \multicolumn{7}{|c|}{ Incidence proportion (\%, 95\% confidence interval) } \\
\hline Anemia, any grade & $89.5(88.6-90.4)$ & $86.3(84.9-87.7)$ & $91.7(89.7-93.8)$ & $98.4(96.7-100)$ & $93.1(91.5-94.8)$ & $93.1(90.3-95.9)$ \\
\hline \multicolumn{7}{|l|}{ Percentage of $\mathrm{CIA}^{\mathrm{a}}$} \\
\hline \multicolumn{7}{|l|}{ Anemia severity } \\
\hline Grade I & 57.8 & 61.0 & 71.4 & 41.1 & 51.3 & 36.4 \\
\hline Grade 2 & 33.7 & 33.3 & 24.1 & 44.7 & 35.3 & 45.5 \\
\hline Grade 3 & 7.6 & 5.3 & 4.2 & II.I & 12.0 & 16.2 \\
\hline Grade 4 & 0.9 & 0.4 & 0.3 & 3.2 & 1.5 & 2.0 \\
\hline \multicolumn{7}{|l|}{ Anemia type } \\
\hline Microcytic & 5.3 & 4.0 & 11.4 & 7.4 & 4.0 & 3.1 \\
\hline Normocytic & 84.9 & 89.3 & 75.9 & 76.7 & 84.6 & 79.6 \\
\hline Macrocytic & 9.8 & 6.7 & 12.7 & 15.9 & 11.4 & 17.3 \\
\hline \multicolumn{7}{|l|}{ Anemia type ${ }^{a}$} \\
\hline Hypochromic & 8.7 & 7.1 & 16.6 & 10.1 & 7.5 & 5.4 \\
\hline Normochromic & 46.9 & 50.7 & 41.8 & 48.1 & 42.8 & 42.5 \\
\hline Hyperchromic & 44.4 & 42.2 & 41.6 & 41.8 & 49.8 & 52.0 \\
\hline
\end{tabular}

Note: a ${ }^{-}$sing the most severe $\mathrm{CIA}$ episode for patients with multiple $\mathrm{CIA}$ episodes.

Abbreviation: $\mathrm{CIA}$, chemotherapy-induced anemia.

Table 3 Distribution of chemotherapy regimens by tumor type

\begin{tabular}{|c|c|c|c|}
\hline \multirow[t]{2}{*}{ Regimen name } & \multirow[t]{2}{*}{$\mathbf{N}(\%)$ of patients } & \multicolumn{2}{|c|}{ Number of cycles } \\
\hline & & Mean (SD) & Median (QI, Q3) \\
\hline \multicolumn{4}{|l|}{ Breast cancer, $n=2,348$} \\
\hline $\mathrm{AC}$ & $109(4.6)$ & $3.5(1)$ & $4(4,4)$ \\
\hline TAC & 157 (6.7) & $5.7(1.0)$ & $6(6,6)$ \\
\hline $\mathrm{AC} \rightarrow \mathrm{T}$ & $655(27.9)$ & $9.2(4.0)$ & $8(8,8)$ \\
\hline Docetaxel + cyclophosphamide (TC) & $855(36.4)$ & $4.5(1.7)$ & $4(4,4)$ \\
\hline $\mathrm{TCH}$ & $399(17.0)$ & $15.9(4.6)$ & $18(16,18)$ \\
\hline Other & $173(7.4)$ & $8.3(6.2)$ & $6(4,13)$ \\
\hline \multicolumn{4}{|l|}{ Colorectal cancer, n=678 } \\
\hline 5-FU with RT & $53(7.8)$ & $4.8(3.2)$ & $6(2,6)$ \\
\hline CAPOX & $155(22.9)$ & $6.5(4.2)$ & $6(3,8)$ \\
\hline FOLFOX & $390(57.5)$ & $10.3(4.7)$ & $12(8,12)$ \\
\hline Other & $80(11.8)$ & $4.8(5.7)$ & $3(2,6)$ \\
\hline \multicolumn{4}{|l|}{ Gastric cancer $n=193$} \\
\hline 5-FU + LV + RT & $43(22.3)$ & $3.3(1.1)$ & $4(3,4)$ \\
\hline CAPOX & $24(12.4)$ & $4.8(2.6)$ & $6(3,7)$ \\
\hline Carboplatin + paclitaxel + RT & $13(6.7)$ & $1.8(1.7)$ & $\mathrm{I}(\mathrm{I}, \mathrm{I})$ \\
\hline ECF & $2(1.0)$ & $3.5(3.5)$ & $4(1,6)$ \\
\hline EOX & $40(20.7)$ & $5.1(2.4)$ & $6(3,6)$ \\
\hline FOLFOX & $25(13.0)$ & $3.7(3.1)$ & $2(2,4)$ \\
\hline Other & $46(23.8)$ & $4.4(2.3)$ & $4(3,6)$ \\
\hline \multicolumn{4}{|l|}{ Lung cancer $n=888$} \\
\hline CARBO + GEM & $89(10.0)$ & $4.0(1.9)$ & $4(3,6)$ \\
\hline CARBO + TAX & $303(34.1)$ & $3.8(2.2)$ & $4(2,6)$ \\
\hline CARBO + VPI6 & $209(23.5)$ & $3.7(1.7)$ & $4(2,5)$ \\
\hline Cisplatin (or carboplatin) + permetrexed & $162(18.2)$ & $5.9(4.7)$ & $4(4,6)$ \\
\hline Cisplatin (or carboplatin) + vinorelbine & $47(5.3)$ & $3.4(1.3)$ & $4(3,4)$ \\
\hline Pemetrexed & $27(3.0)$ & $5.8(5.0)$ & $4(2, I I)$ \\
\hline Other & $5 \mathrm{I}(5.7)$ & $4.0(3.8)$ & $3(1,6)$ \\
\hline \multicolumn{4}{|l|}{ Ovarian cancer $n=319$} \\
\hline CARBO + TAX & 293 (91.9) & $6.0(2.3)$ & $6(6,6)$ \\
\hline Carboplatin (or cisplatin) only \pm bevacitumab & $14(4.4)$ & $6.9(4.5)$ & $6(6,6)$ \\
\hline Other & $12(3.8)$ & $4.2(1.8)$ & $4(3,6)$ \\
\hline
\end{tabular}

Abbreviations: AC, Doxorubicin + cyclophosphamide; TAC, Docetaxel + doxorubicin + cyclophosphamide; AC $\rightarrow$ T, Doxorubicin + cyclophosphamide followed by paclitaxel or docetaxel; TCH, Docetaxel + carboplatin + trastuzumab; 5-FU, 5-fluorouracil; RT, radiation therapy; CAPOX, Capecitabine + oxaliplatin; FOLFOX, Leucovorin calcium + 5-fluorouracil + oxaliplatin; 5-FU + LV + RT, 5-Fluorouracil + leucovorin + radiation therapy; ECF, Epirubicin + cisplatin + 5-fluorouracil; EOX, Epirubicin + oxaliplatin + capecitabine; CARBO + GEM, Carboplatin + gemcitabine; CARBO + TAX, Carboplatin + paclitaxel; CARBO + VPI6, Carboplatin + etoposide; SD, standard deviation. 
Table 4 Incidence proportion of anemia associated with selected regimens

\begin{tabular}{|c|c|c|c|c|c|}
\hline \multirow[t]{2}{*}{ Regimen name } & \multirow{2}{*}{$\begin{array}{l}\text { Incidence }(\%, 95 \% \mathrm{Cl}) \\
\text { Any grade }\end{array}$} & \multicolumn{4}{|c|}{ Percentage of CIA (\%) } \\
\hline & & Grade I & Grade II & Grade III & Grade IV \\
\hline \multicolumn{6}{|l|}{ Breast cancer } \\
\hline $\mathrm{AC}$ & $78.9(71.2-86.6)$ & 57.0 & 34.9 & 8.1 & 0 \\
\hline TAC & 91.1 (86.6-95.5) & 60.8 & 35.7 & 2.8 & 0.7 \\
\hline $\mathrm{AC} \rightarrow \mathrm{T}$ & $95.7(94.2-97.3)$ & 57.3 & 38.0 & 4.5 & 0.3 \\
\hline $\mathrm{TC}$ & 77.1 (74.3-79.9) & 76.3 & 20.6 & 2.6 & 0.5 \\
\hline $\mathrm{TCH}$ & $96.2(94.4-98.1)$ & 43.8 & 45.3 & 10.7 & 0.3 \\
\hline \multicolumn{6}{|l|}{ Colorectal cancer } \\
\hline CAPOX & $89.7(84.9-94.5)$ & 72.7 & 24.5 & 2.9 & 0 \\
\hline FOLFOX & $94.6(92.4-96.9)$ & 73.7 & 21.7 & 4.3 & 0.3 \\
\hline \multicolumn{6}{|l|}{ Lung cancer } \\
\hline CARBO + TAX & 93.1 (90.2-95.9) & 59.6 & 31.2 & 9.2 & 0 \\
\hline CARBO + VPI6 & $94.7(91.7-97.8)$ & 42.4 & 41.9 & 13.6 & 2.0 \\
\hline Cisplatin (or carboplatin) + permetrexed & $86.4(8||-9 \mid .7)$. & 57.1 & 27.9 & 12.9 & 2.1 \\
\hline \multicolumn{6}{|l|}{ Ovarian cancer } \\
\hline CARBO + TAX & $92.8(89.9-95.8)$ & 35.7 & 46.0 & 16.2 & 2.2 \\
\hline
\end{tabular}

Notes: ${ }^{2} U$ sing the most severe $\mathrm{CIA}$ episode for patients with multiple $\mathrm{ClA}$ episodes.

Abbreviations: AC, Doxorubicin + cyclophosphamide; TAC, Docetaxel + doxorubicin + cyclophosphamide; AC $\rightarrow$ T, Doxorubicin + cyclophosphamide followed by paclitaxel or docetaxel; TC, Docetaxel + cyclophosphamide; TCH, Docetaxel + carboplatin + trastuzumab; CAPOX, Capecitabine + oxaliplatin; FOLFOX, Leucovorin calcium + 5-fluorouracil + oxaliplatin; CARBO + TAX, Carboplatin + paclitaxel; CARBO + VPI6, Carboplatin + etoposide; Cl, confidence interval; CIA, chemotherapy-induced anemia.

Institute, Bethesda, MD, USA) regimen. A smaller incidence proportion of patients had grade $3+$ anemia $(\mathrm{Hb}<8 \mathrm{~g} / \mathrm{dL})$, with the lowest proportion in $2 \%$ breast cancer patients treated with TC regimen and the largest proportion (17\%) in ovarian cancer patients receiving Carbo-Tax (Table 4, Figure 2). Across cancers, our analysis showed that the incidence of any grade anemia increased from $59 \%$ in cycle 1 to $83 \%$ by cycle 5 . We also observed a steady increase in the fraction of grades 2 or 3 anemia associated with a great number of cycles. Figure 3 shows the incidence of anemia and the fractions in each grade for selected regimens by chemotherapy cycles.
We repeated the analyses by cancer stage. The incidence of anemia was greater in patients with advanced stages. Overall, the risk of grade $2+$ anemia was increased from $29 \%$ in stage I to $49 \%$ in stage IV. The incidence of grade 3 or 4 anemia was approximately $12 \%$ in patients with stage IV disease, ranging from $3.7 \%$ in patients with stage IV colorectal cancer to $21.2 \%$ in patients with stage IV ovarian cancer (Figure 4).

\section{Discussion}

Anemia is a common hematologic complication of chemotherapy, and is associated with increased morbidity, mortality,

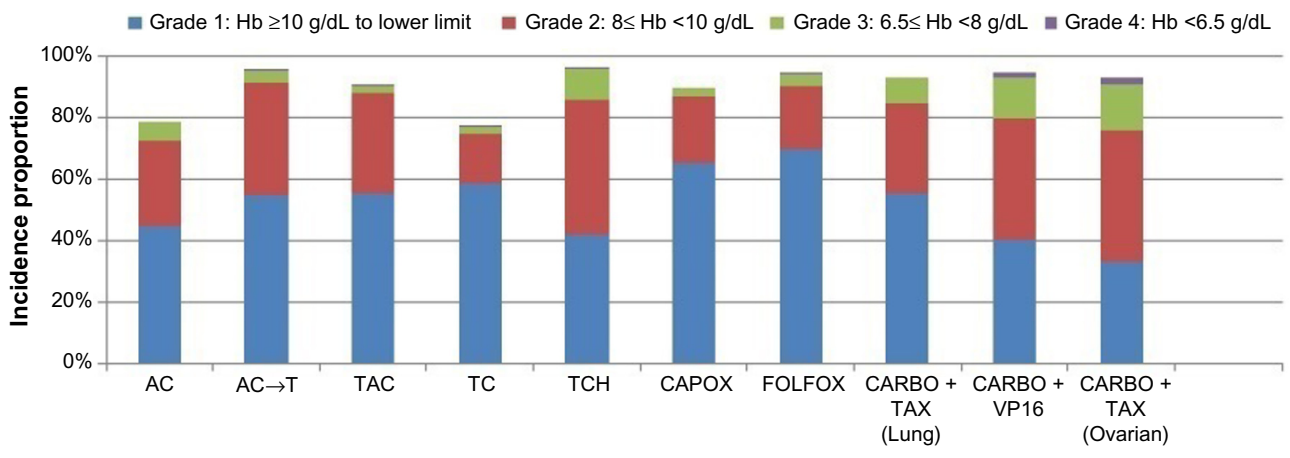

Figure 2 Incidence proportion of anemia by grade associated with selected regimens during the course of chemotherapy.

Abbreviations: AC, doxorubicin (Adriamycin) and cyclophosphamide (Cytoxan); TAC, docetaxel (Taxotere), doxorubicin (Adriamycin), and cyclophosphamide (Cytoxan); $\mathrm{AC} \rightarrow \mathrm{T}$, doxorubicin (Adriamycin) and cyclophosphamide (Cytoxan) followed by paclitaxel (Taxol) or docetaxel (Taxotere); TC, docetaxel (Taxotere) and cyclophosphamide (Cytoxan); TCH, Taxotere, Carboplatin, Herceptin; CAPOX, capecitabine and oxaliplatin; FOLFOX, Leucovorin calcium, 5-Fluorouracil and Oxaliplatin; CARBO + TAX, carboplatin and Paclitaxel (Taxol); CARBO + VPI6, Carboplatin and Etoposide; Hb, hemoglobin. 

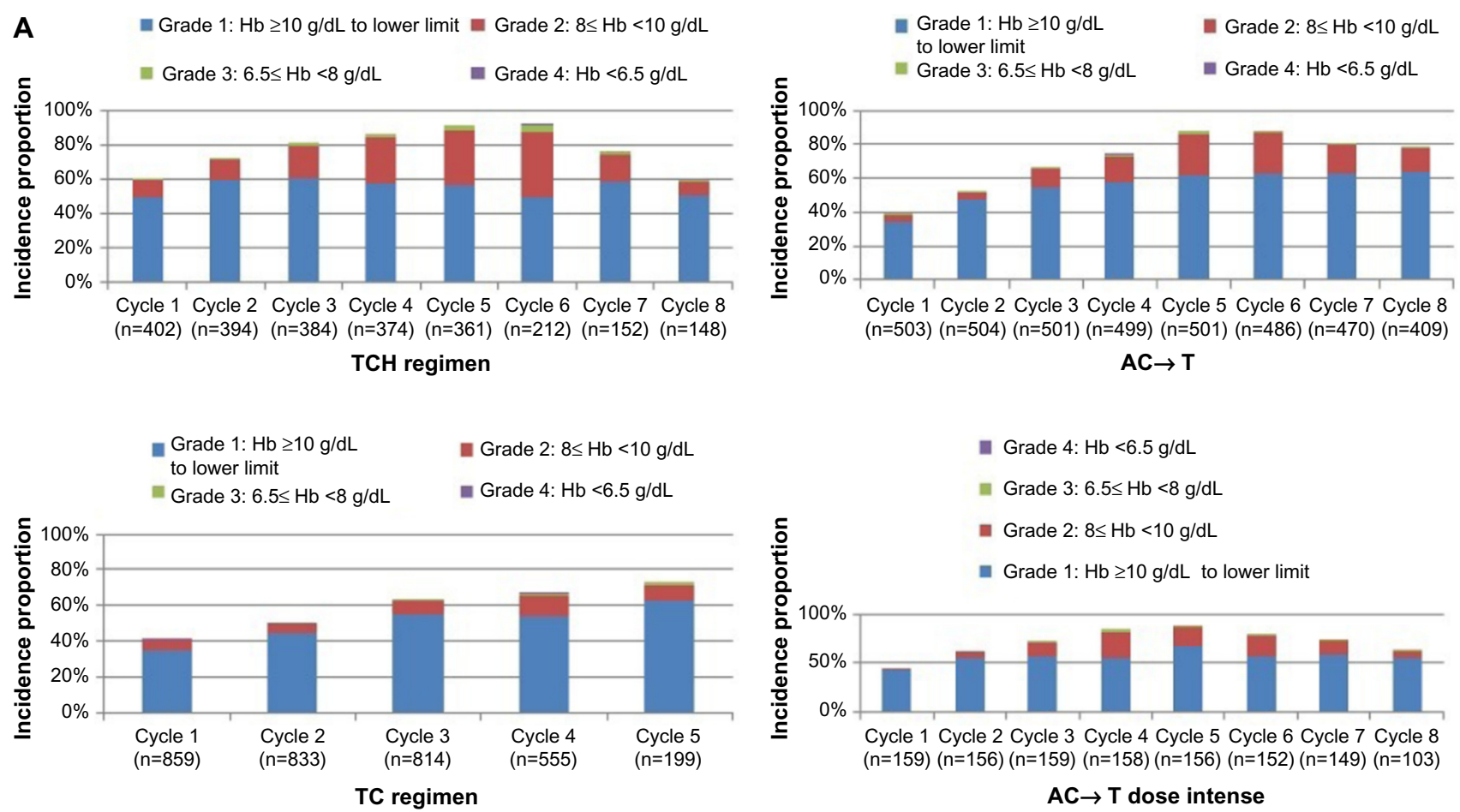

B

Grade 1: $\mathrm{Hb} \geq 10 \mathrm{~g} / \mathrm{dL}$ to lower limit

Grade $2: 8 \leq \mathrm{Hb}<10 \mathrm{~g} / \mathrm{dL}$

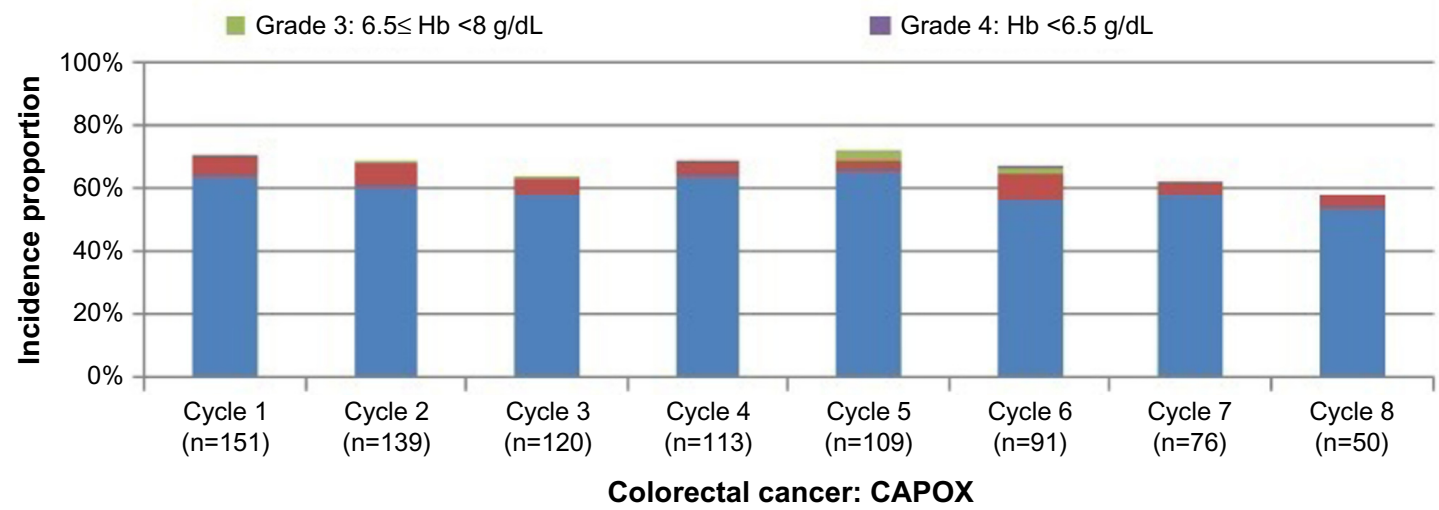

\section{Colorectal cancer: CAPOX}

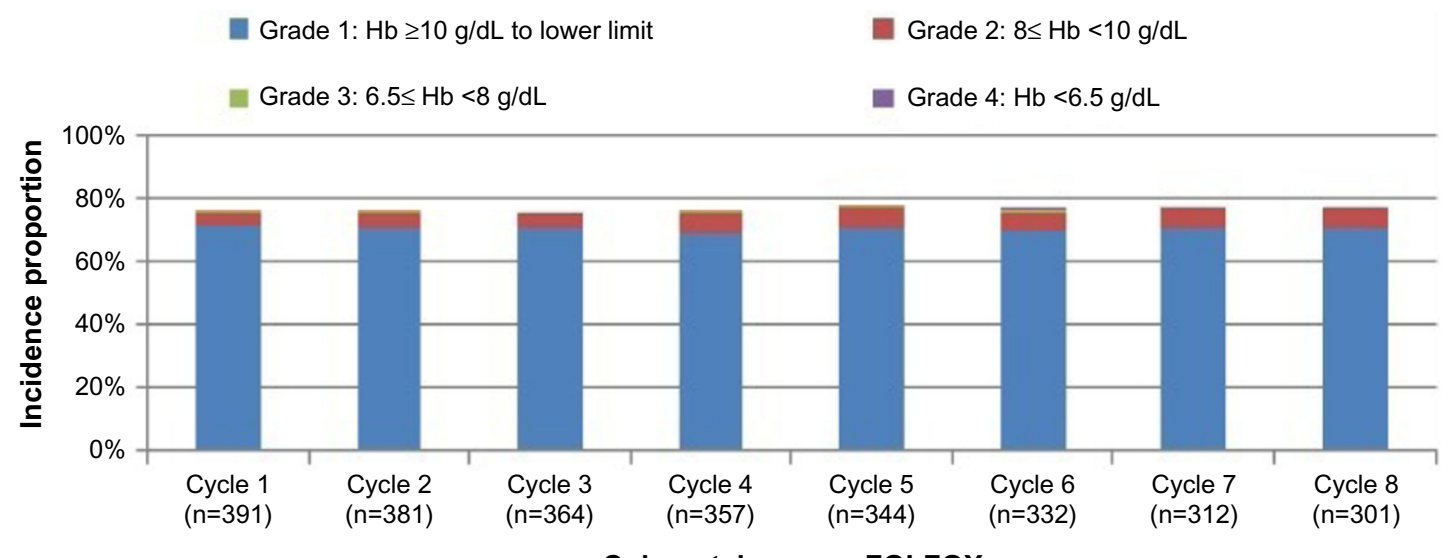

Colorectal cancer: FOLFOX

Figure 3 (Continued) 


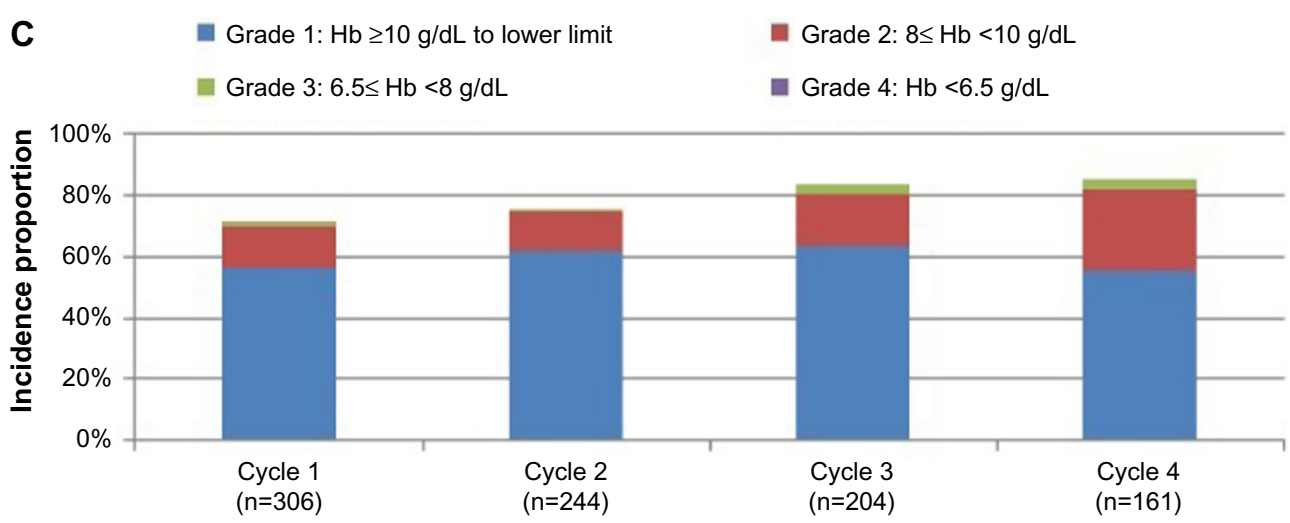

Lung cancer: CARBO + TAX

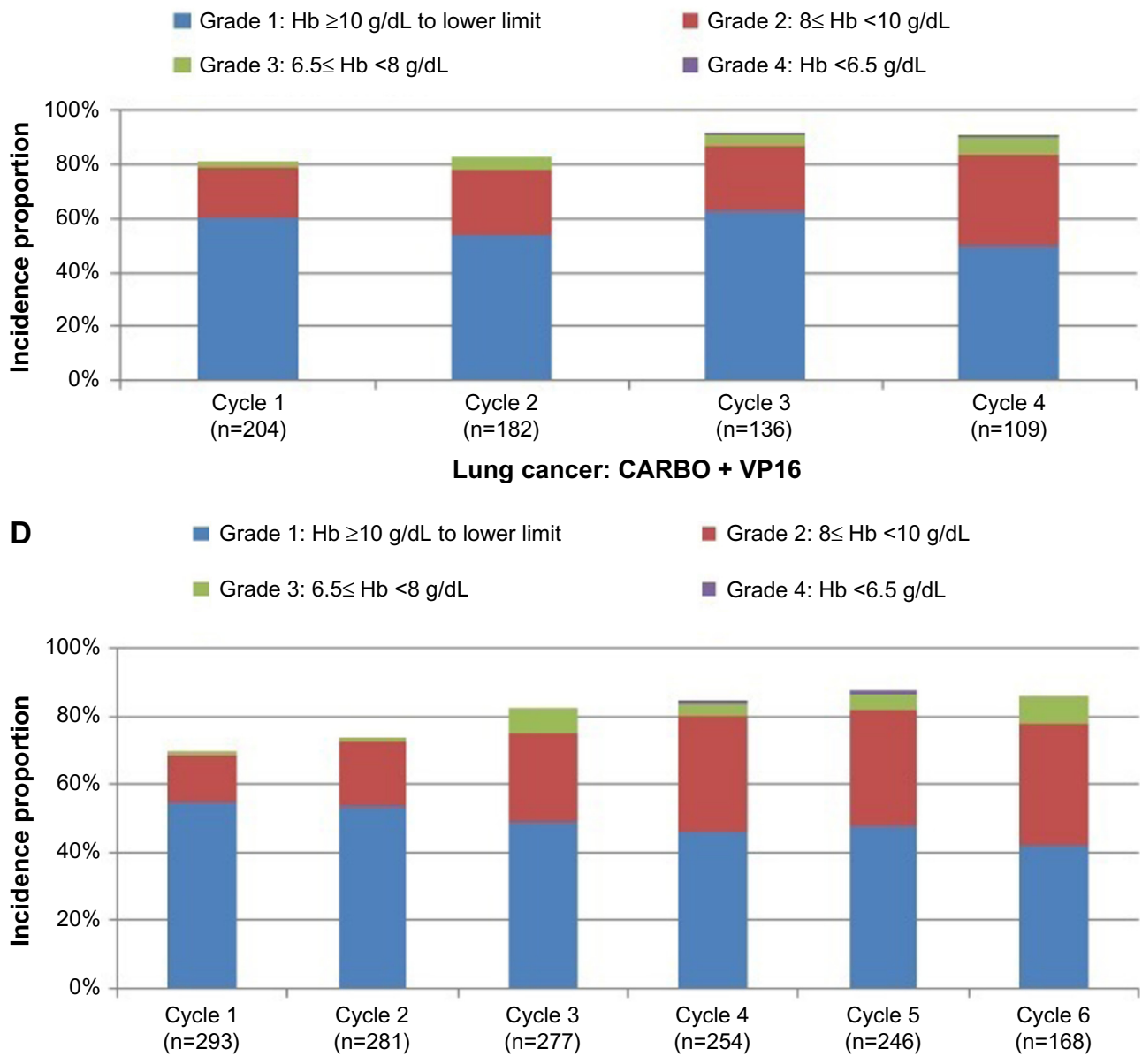

Ovarian cancer: CARBO + TAX

Figure 3 Incidence of anemia among patients with four common cancers.

Notes: (A) Incidence of anemia by chemotherapy regimens and cycles among patients with breast cancer, (B) colorectal cancer, (C) lung cancer, and (D) ovarian cancer. Abbreviations: AC to T, doxorubicin (Adriamycin) and cyclophosphamide (Cytoxan) followed by paclitaxel (Taxol) or docetaxel (Taxotere); TC, docetaxel (Taxotere) and cyclophosphamide (Cytoxan); TCH, Taxotere, Carboplatin, Herceptin; CAPOX, capecitabine and oxaliplatin; FOLFOX, Leucovorin calcium, Fluorouracil and Oxaliplatin; CARBO + TAX, carboplatin and Paclitaxel (Taxol); CARBO + VPI6, Carboplatin and Etoposide; Hb, hemoglobin.

and health-care cost. Although incidence and prevalence of CIA have been extensively reported in previous studies, ${ }^{1,4,5,7}$ little data exist on the risk of anemia associated with chemotherapy regimens in current oncology practice. This retrospective cohort study, using data from the large integrated KPSC Health Plan, examined and characterized the risk of anemia among patients with solid tumors receiving chemotherapy. We found that the risk of moderate-to-severe CIA $(\mathrm{Hb}<10 \mathrm{~g} / \mathrm{dL})$ remained considerably high (38\%) in patients with five common types of solid tumors receiving 

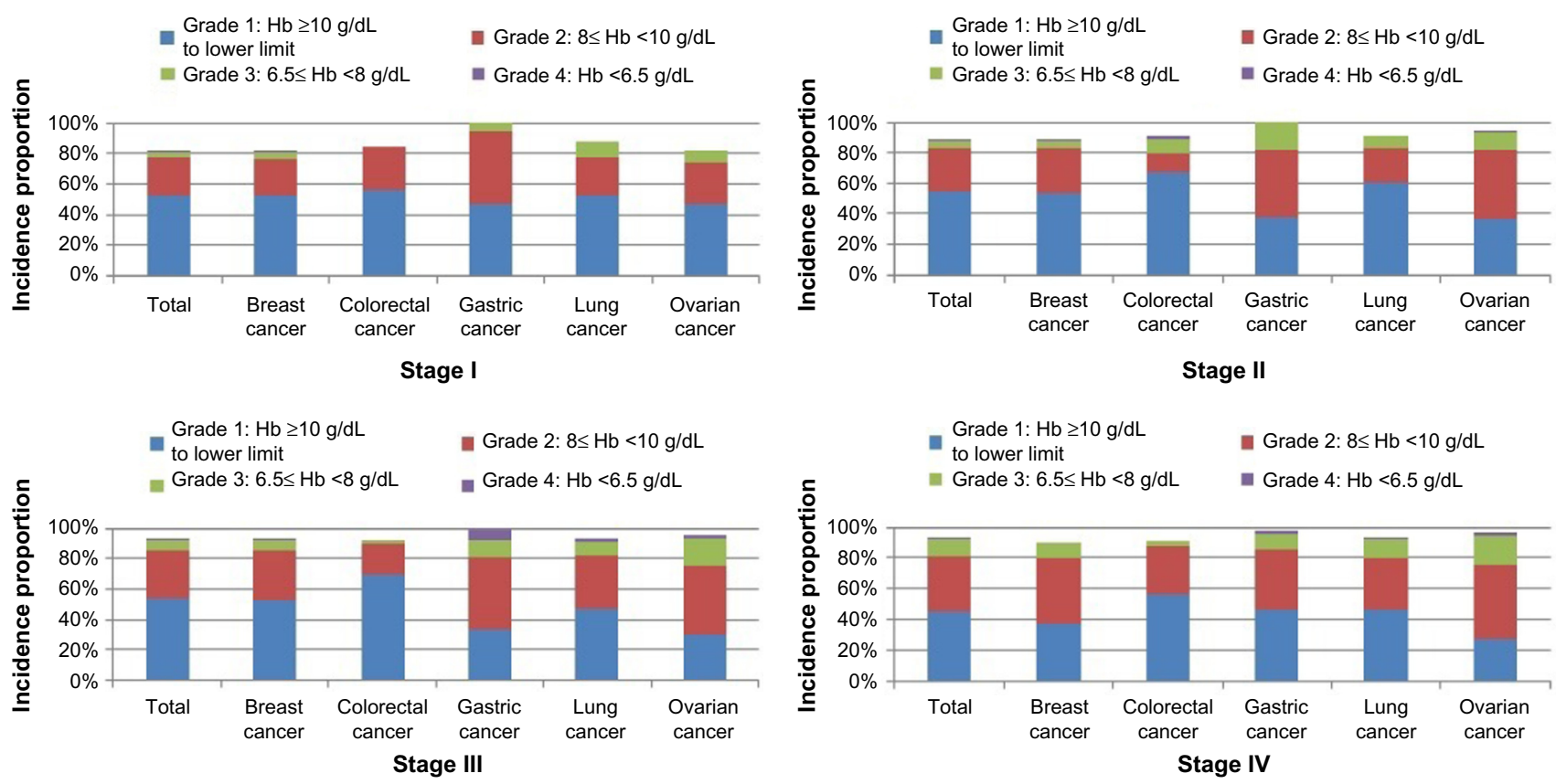

Figure $\mathbf{4}$ Incidence proportion of anemia among cancer patients receiving chemotherapy by stage. Abbreviation: $\mathrm{Hb}$, hemoglobin.

chemotherapy, ranging from $26 \%$ in colorectal cancer to $59 \%$ in ovarian cancer; and the risk of anemia was even greater in patients with more advanced cancer. The incidence of severe anemia $(\mathrm{Hb}<8 \mathrm{~g} / \mathrm{dL})$ was highest in patients with ovarian cancer $(16.9 \%)$ and lowest in patients with colorectal cancer (4\%). The incidence of anemia varied significantly across chemotherapy regimens, with the proportions of patients with $\mathrm{Hb}<10 \mathrm{~g} / \mathrm{dL}$ ranging from $18.2 \%$ in breast cancer patients treated with TC regimen to $59.7 \%$ in patients with ovarian cancer receiving Carbo-Tax regimen. We also observed a steady increase in the incidence of anemia as well as the increased fractions of grades 2-4 anemia up to cycle 5, and the incidence declined slightly afterward.

The incidence of anemia in cancer patients reported in the previous studies varies significantly, which may be due to variations in patient characteristics, disease and treatment characteristics, as well as differences in the study methods, including in anemia definitions. ${ }^{4,5,7}$ For instance, a large prospective The European Cancer Anaemia Survey (ECAS) reported that the rate of anemia (defined as $\mathrm{Hb}<12 \mathrm{~g} / \mathrm{dL}$ ) was $75 \%$ in patients treated with chemotherapy, compared with $40 \%$ in those not undergoing chemotherapy. ${ }^{7}$ In another study using a large outpatient oncology electronic medical record database, the rate of anemia (defined as $\mathrm{Hb}<11 \mathrm{~g} / \mathrm{dL}$ ) was $20.9 \%$ at baseline and increased to $46.4-59.0 \%$ after treatment with chemotherapy regimens. ${ }^{4}$ In addition, the proportion of patients with $\mathrm{Hb}<10 \mathrm{~g} / \mathrm{L}$ was largest in those treated with gemcitabine-based regimen $(26.7 \%)$, followed by platinum-based regimen $(20.8 \%)$, anthracycline-based regimen (19.5\%), taxane-based regimen (18.7\%), and other regimens $(16.2 \%) .{ }^{4}$ Most of the previous studies included patients with anemia at baseline in the analysis, while our study excluded patients who had a diagnosis of anemia of other causes or had an $\mathrm{Hb}<10 \mathrm{~g} / \mathrm{dL}$ prior to chemotherapy initiation. Compared to previous studies, we observed a relatively higher proportion of patients with moderate or severe anemia in current oncology practice, greatest in patients with ovarian cancer. Despite the differences in the study population, study design, and methods of analysis, it is possible that the potential shift in the perceived $\mathrm{Hb}$ trigger level for anemia treatment has resulted in an overall increase in the risk and the severity of anemia due to recent declines in ESA use.

We observed a steady increase in the incidence of anemia up to cycle 5 or 6 , and the incidence declined slightly afterward. The delayed mode likely reflects the natural life span of the human RBC, which is approximately 120 days. ${ }^{18}$ Risk increases over time also due to the accumulated myelosuppressive effects of cytotoxic agents over the repeated cycles of therapy. The later decline in risk is likely due to the combined effects of changes in chemotherapy (dose delays, dose reductions, and changed regimens), anemia treatments, and potentially selective losses to follow-up.

It should be noted that the etiology of anemia is multifactorial. In this study, we found that approximately $10 \%$ 
of anemic patients were either microcytic or macrocytic; and around $10 \%$ of patients were hypochromic. A comprehensive evaluation of anemia as well as the underlying conditions, such as the nutritional status and bone marrow function, may help guide anemia management. There were significant variations in the risk and severity of anemia across chemotherapy regimens. An increased surveillance and more proactive treatment strategy may be needed for those patients receiving "high-risk" regimens (eg, Carbo-Tax).

Our studies have several strengths. Published data on the anemia risk profile available in the literature are relatively old, and new data are needed given recent changes in both chemotherapy practice and anemia management. To our knowledge, this is the first comprehensive evaluation of anemia risk, including both anemia severity (grade) and morphological type, among large cohorts of patients receiving chemotherapy regimens in current US practice. Evidence on the risk of anemia associated with some emerging regimens (eg, $\mathrm{TC}, \mathrm{TCH})$ is available from clinical trials. Such evidence, however, may not be reflective of clinical practice, because patients and their treatments may vary greatly in this setting (versus those in an experimental setting). Patients in clinical practice are likely to differ from trial subjects in significant ways (eg, more comorbidities, older age, and poorer performance status) that would render them at higher risk of anemia. In addition, other factors such as use of ESAs, and chemotherapy dose delays and dose reductions - may lead to variation in the anemia profile of patients between the clinical trial and clinical practice settings.

This retrospective study had some limitations worthy of comment. First, the data were extracted from electronic medical records rather than being prospectively collected. It is possible that some anemias may go underdiagnosed due to lack of tests ordered. We were not able to further characterize the reasons for anemia due to lack of information on specific tests, such as measures of iron, folate, and $B_{12}$ status. The study analyses focused primarily on the incidence, severity, and type of anemia associated with different chemotherapy regimens; however, it did not take into account coexisting illness, treatment history, and changes in treatment including chemotherapy, which may have had an impact on the risk and the severity of anemia assessed. In addition, our findings were based on a practice in a highly integrated managed care organization. Despite the fact that the KPSC population is broadly representative of the southern California population at large, our findings may not be generalizable to all US cancer patients, particularly those without health insurance, or patients managed in other practice types.

In summary, the results of this study suggested that the risk of anemia remained high among patients with solid tumors receiving chemotherapy. The risk of anemia was greater in patients with distant metastasis. The incidence of anemia varied significantly by chemotherapy regimen and steadily increased over cycles. Patients with anemia should be closely monitored, and a vigilant management strategy may be implemented to reduce the risk of morbidity associated with anemia.

\section{Acknowledgment}

Funding for this research was provided by Amgen Inc. to Kaiser Permanente Southern California.

\section{Disclosure}

Hairong $\mathrm{Xu}$ and John $\mathrm{H}$ Page are employed by Amgen Inc. Chun Chao, Lanfang Xu, Kim Cannavale, Olivia Sattayapiwat, and Roberto Rodriguez are employees of Kaiser Permanente Southern California. The authors report no other conflicts of interest in this work.

\section{References}

1. Schwartz RN. Anemia in patients with cancer: incidence, causes, impact, management, and use of treatment guidelines and protocols. Am J Health Syst Pharm. 2007;64(3 Suppl 2):S5-S13; quiz S28-S30.

2. Rodgers GM 3rd, Becker PS, Blinder M, et al. Cancer- and chemotherapyinduced anemia. J Natl Compr Canc Netw. 2012;10(5):628-653.

3. Dicato M, Plawny L, Diederich M. Anemia in cancer. Ann Oncol. 2010;21(Suppl 7):vii167-vii172.

4. Wu Y, Aravind S, Ranganathan G, Martin A, Nalysnyk L. Anemia and thrombocytopenia in patients undergoing chemotherapy for solid tumors: a descriptive study of a large outpatient oncology practice database, 2000-2007. Clin Ther. 2009;31(Pt 2):2416-2432.

5. Groopman JE, Itri LM. Chemotherapy-induced anemia in adults: incidence and treatment. J Natl Cancer Inst. 1999;91(19):1616-1634.

6. Capo G, Waltzman R. Managing hematologic toxicities. J Support Oncol. 2004;2(1):65-79.

7. Ludwig H, Van Belle S, Barrett-Lee P, et al. The European Cancer Anaemia Survey (ECAS): a large, multinational, prospective survey defining the prevalence, incidence, and treatment of anaemia in cancer patients. Eur J Cancer. 2004;40(15):2293-2306.

8. Harper P, Littlewood T. Anaemia of cancer: impact on patient fatigue and long-term outcome. Oncology. 2005;69(Suppl 2):2-7.

9. Nieboer P, Buijs C, Rodenhuis S, et al. Fatigue and relating factors in high-risk breast cancer patients treated with adjuvant standard or high-dose chemotherapy: a longitudinal study. J Clin Oncol. 2005;23(33):8296-8304.

10. Cella D, Kallich J, McDermott A, Xu X. The longitudinal relationship of hemoglobin, fatigue and quality of life in anemic cancer patients: results from five randomized clinical trials. Ann Oncol. 2004;15(6):979-986. 
11. Leyland-Jones B, Semiglazov V, Pawlicki M, et al. Maintaining normal hemoglobin levels with epoetin $\alpha$ in mainly nonanemic patients with metastatic breast cancer receiving first-line chemotherapy: a survival study. J Clin Oncol. 2005;23(25):5960-5972.

12. Henke M, Laszig R, Rube C, et al. Erythropoietin to treat head and neck cancer patients with anaemia undergoing radiotherapy: randomised, double-blind, placebo-controlled trial. Lancet. 2003; 362(9392):1255-1260.

13. Smith RE Jr, Aapro MS, Ludwig H, et al. Darbepoetin $\alpha$ for the treatment of anemia in patients with active cancer not receiving chemotherapy or radiotherapy: results of a phase III, multicenter, randomized, double-blind, placebo-controlled study. J Clin Oncol. 2008;26(7):1040-1050.

14. Wright JR, Ung YC, Julian JA, et al. Randomized, double-blind, placebocontrolled trial of erythropoietin in non-small-cell lung cancer with disease-related anemia. J Clin Oncol. 2007;25(9):1027-1032.
15. Juneja V, Keegan P, Gootenberg JE, et al. Continuing reassessment of the risks of erythropoiesis-stimulating agents in patients with cancer. Clin Cancer Res. 2008;14(11):3242-3247.

16. Xu H, Kaye JA, Saltus CW, Crawford J, Gasal E, Goodnough LT. Blood utilization and hemoglobin levels in cancer patients after label and coverage changes for erythropoiesis-stimulating agents. Expert Rev Hematol. 2014;7(5):617-633.

17. Koebnick C, Langer-Gould AM, Gould MK, et al. Sociodemographic characteristics of members of a large, integrated health care system comparison with US Census Bureau data. Perm J. 2012;16(3):37-41.

18. Shemin D, Rittenberg D. The life span of the human red blood cell J Biol Chem. 1946;166(2):627-636.
Clinical Epidemiology

\section{Publish your work in this journal}

Clinical Epidemiology is an international, peer-reviewed, open access, online journal focusing on disease and drug epidemiology, identification of risk factors and screening procedures to develop optimal preventative initiatives and programs. Specific topics include: diagnosis, prognosis, treatment, screening, prevention, risk factor modification,

Submit your manuscript here: http://www.dovepress.com/clinical-epidemiology-journa

\section{Dovepress}

systematic reviews, risk \& safety of medical interventions, epidemiology \& biostatistical methods, and evaluation of guidelines, translational medicine, health policies \& economic evaluations. The manuscript management system is completely online and includes a very quick and fair peer-review system, which is all easy to use. 